\title{
Desmoid-type fibromatosis of the breast: A report of 2 cases
}

\author{
ROMAN BENEJ ${ }^{1}$, IVETA MEČIAROVÁ ${ }^{2}$ and KAMIL POHLODEK ${ }^{3}$ \\ ${ }^{1}$ Department of Thoracic Surgery, Slovak Medical University of Bratislava, 82606 Bratislava; \\ ${ }^{2}$ Alpha Medical Pathology, Ltd., 84101 Bratislava; ${ }^{3}$ Second Department of Gynecology and Obstetrics, \\ Comenius University of Bratislava, Faculty of Medicine, 82606 Bratislava, Slovakia
}

Received February 15, 2017; Accepted May 8, 2017

DOI: $10.3892 / 01.2017 .6337$

\begin{abstract}
Mammary fibromatosis is a rare and locally aggressive benign tumor of the breast; it originates from fibroblasts and myofibroblasts within the breast parenchyma and does not metastasize. The condition is locally aggressive and has a high rate of recurrence. The etiology of mammary fibromatosis is unknown. Breast imaging examinations are not specific for fibromatosis and often imitate breast cancer. The current study presents 2 cases of women with breast fibromatosis, the first of which exhibited a locally advanced aggressive form of the disease, where breast surgery and en bloc resection of the underlying regions of the thoracic wall were required. In the second case, breast imaging examinations suggested an invasive breast tumor, probably carcinoma, infiltrating the muscles of the chest wall. An ultrasound-guided core needle biopsy revealed a low-grade myofibroblastic proliferation consistent with breast fibromatosis. The patient underwent a right quadrantectomy, with a partial resection of the underlying musculature. The patients remain disease-free at the time of writing. As involvement of the breast in patients with desmoid-like fibromatosis as rare, the present study reports 2 cases with clinical features and histological findings in order to improve and add to the knowledge of this disease.
\end{abstract}

\section{Introduction}

Desmoid-type fibromatosis, previously described as aggressive fibromatosis, is a rare soft tissue tumor with the typical clinical behavior of frequent local recurrence without distant spread (1). It usually occurs in the abdominal wall and in the superficial muscular-aponeurotic tissues of the extremities (1-3). Mammary desmoid-type fibromatosis is a rare and locally aggressive intermediate tumor of the breast; it originates from fibroblasts and myofibroblasts within the breast parenchyma and does not metastasize (1-5). The condition is locally

Correspondence to: Dr Kamil Pohlodek, Second Department of Gynecology and Obstetrics, Comenius University of Bratislava, Faculty of Medicine, Ružinovská 6, 82606 Bratislava, Slovakia E-mail:kpohlodek@gmail.com

Key words: breast tumors, desmoid-type fibromatosis aggressive and has a high rate of recurrence. Extra-abdominal desmoid tumors account for $3 \%$ of all soft-tissue tumors, with an incidence of 2.4-4.3 per million each year (1). Fibromatosis of the chest wall represents 8-10\% of all cases (2). Fibromatosis arising from within the breast itself is very rare, accounting for $0.2 \%$ of all breast tumors (5-12). The etiology of mammary fibromatosis is unknown, and breast imaging examinations are not specific for fibromatosis; to distinguish mammary fibromatosis from malignant breast tumors through physical examination and imaging techniques can be difficult. There appears to be general agreement that a complete wide excision is the treatment of choice of fibromatosis involving only the breast (1-4). The current study presents 2 cases of women with breast fibromatosis, including one with a locally advanced aggressive form of the disease.

\section{Case reports}

Case 1. A 36-year-old Caucasian woman with no previous medical history was referred in September 2016 to the Breast Unit of the Second Department of Gynecology and Obstetrics, University Hospital of Bratislava (Slovakia), for a rapidly growing lump in the right breast following a small trauma. Physical examination revealed a large, breast- and skin-infiltrating, prominent tumor located predominantly in the lateral quadrants of the right breast (Fig. 1A). The tumor mass was fixed to the chest wall. A well circumscribed hypoechoic area with a rather homogenous echotexture and lateral acoustic shadowing could be observed on breast ultrasound (Fig. 1B). Mammography suggested a circumscribed, round, radiopaque lesion with mostly sharp contours, with the dorsal region of the lesion being impossible to define (Fig. 1C). As the tumor was fixed to the chest wall, a thoracoabdominal computed tomography scan was made; it showed a large $(8.4 \times 7.5 \times 5.4 \mathrm{~cm})$, inhomogeneous mass in the right breast that appeared to be in continuity with the right pectoralis major muscle and the right serratus anterior muscle (Fig. 2). Findings on the intra-thoracic and intra-abdominal organs were physiological. A core needle biopsy was performed on the palpable right breast mass, which supposed the diagnosis of fibromatosis. Once a diagnosis was established, surgery was scheduled. The patient was taken to the operating room at the Department of Thoracic Surgery (University Hospital of Bratislava) for the breast surgery and possible en bloc resection of the underlying regions of the thoracic wall. Surgery was performed under general anesthesia 


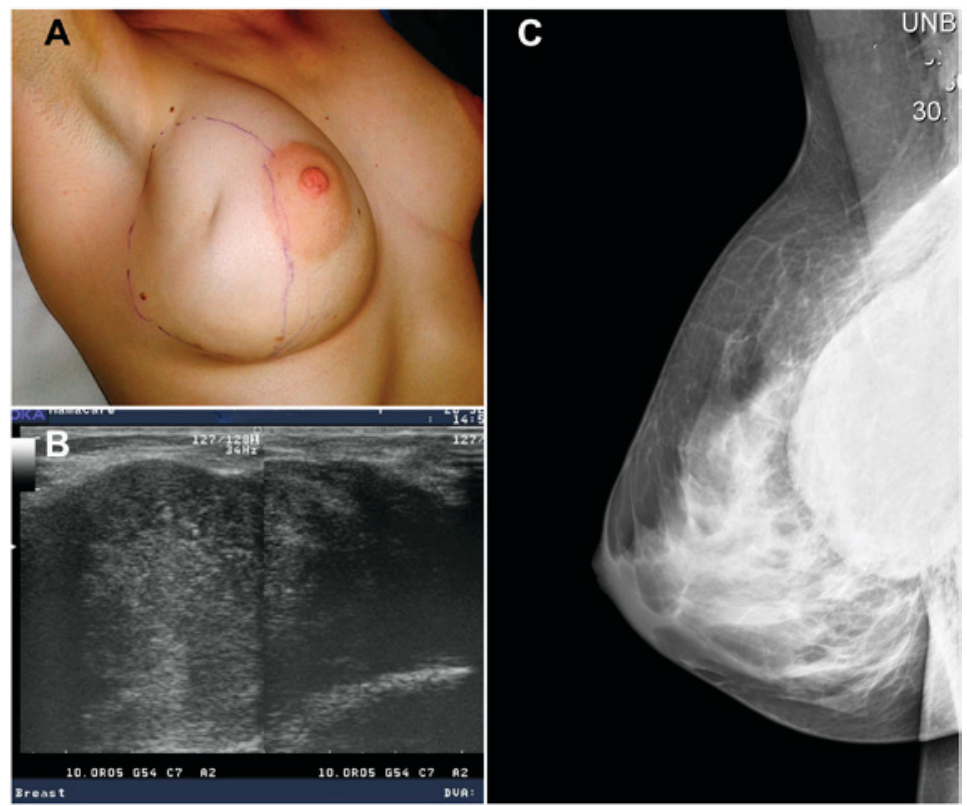

Figure 1. Breast fibromatosis in case 1. (A) Anatomical location of a large palpable mass in the right breast with retracted skin. (B) A well-defined hypoechoic area with non-homogenous echotexture on breast ultrasound. (C) A circumscribed radiopaque lesion with a mostly sharp contour and indeterminate dorsal demarcation in X-ray digital mammography.

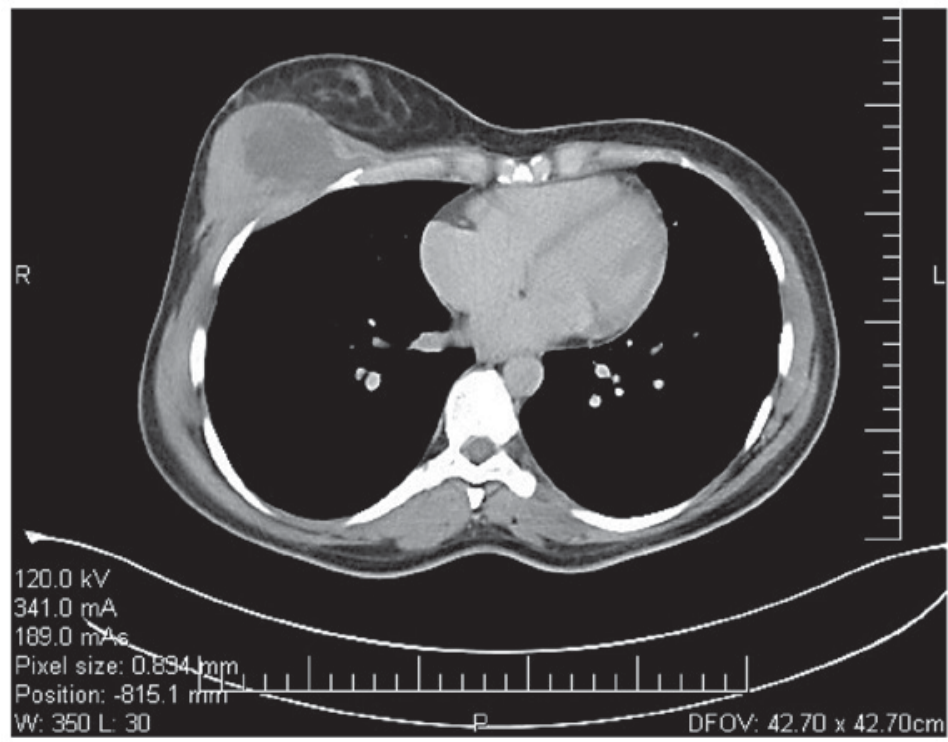

Figure 2. Breast fibromatosis in case 1. Computed tomography thoracic scan in the axial view showing a large tumor mass in the right breast involving the chest wall.

with selective intubation. The patient underwent a right partial mastectomy with en bloc resection of the underlying musculature (inferior lateral portion of the right pectoralis major muscle and the anterior portion of the right serratus anterior muscle) and en bloc resection of the underlying chest wall structures (fourth and fifth ribs, intercostal muscles and parietal pleura; Fig. 3A). Repeated frozen section examinations during surgery were required for confirmation or exclusion of chest wall infiltration. The right chest wall defect was then closed with a $15.0 \times 15.0-\mathrm{cm}$ polypropylene flat sheet mesh (Parietene $^{\mathrm{TM}}$; Medtronic, Minneapolis, MN, USA; Fig. 3B). The right partial mastectomy site was then closed in a standard manner. No attempts at cosmetic breast reconstruction with autologous tissue transfer or expander/implant placement were considered at that time.

The surgically removed specimen was $12.0 \times 10.5 \times 7.2 \mathrm{~cm}$ in size, containing a grossly circumscribed firm tumor with off-white, whorled patterns, without necrosis (Fig. 4A). The specimen was fixed in $10 \%$ neutral buffered formalin for $24 \mathrm{~h}$. The fixed specimen was trimmed with a scalpel to enable it to fit into a tissue cassette. It was processed in an automated tissue processing machine (BenchMark XT; Ventana Medical Systems, Inc., Tucson, AZ, USA). The processing included dehydration, clearing and embedding, in which the specimens were infiltrated with paraffin wax to create paraffin blocks. These blocks were cut with a rotary microtome (Leica 

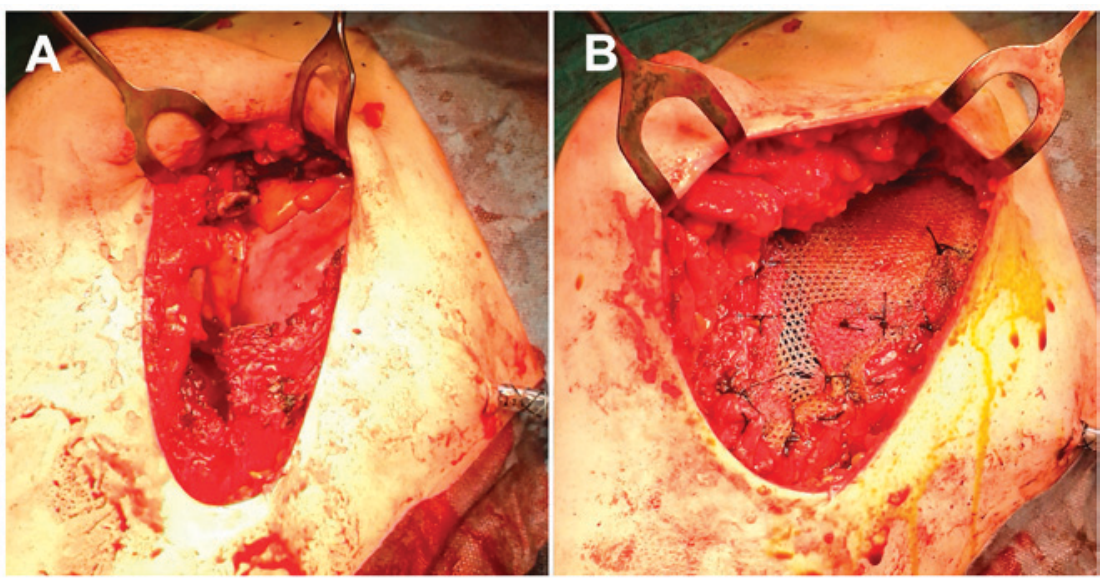

Figure 3. Breast fibromatosis in case 1. (A) Right chest wall defect created by the en bloc resection of the underlying chest wall structures (fourth and fifth ribs, intercostal muscles and parietal pleura). (B) The defect was then closed with a flat sheet of polypropylene mesh.

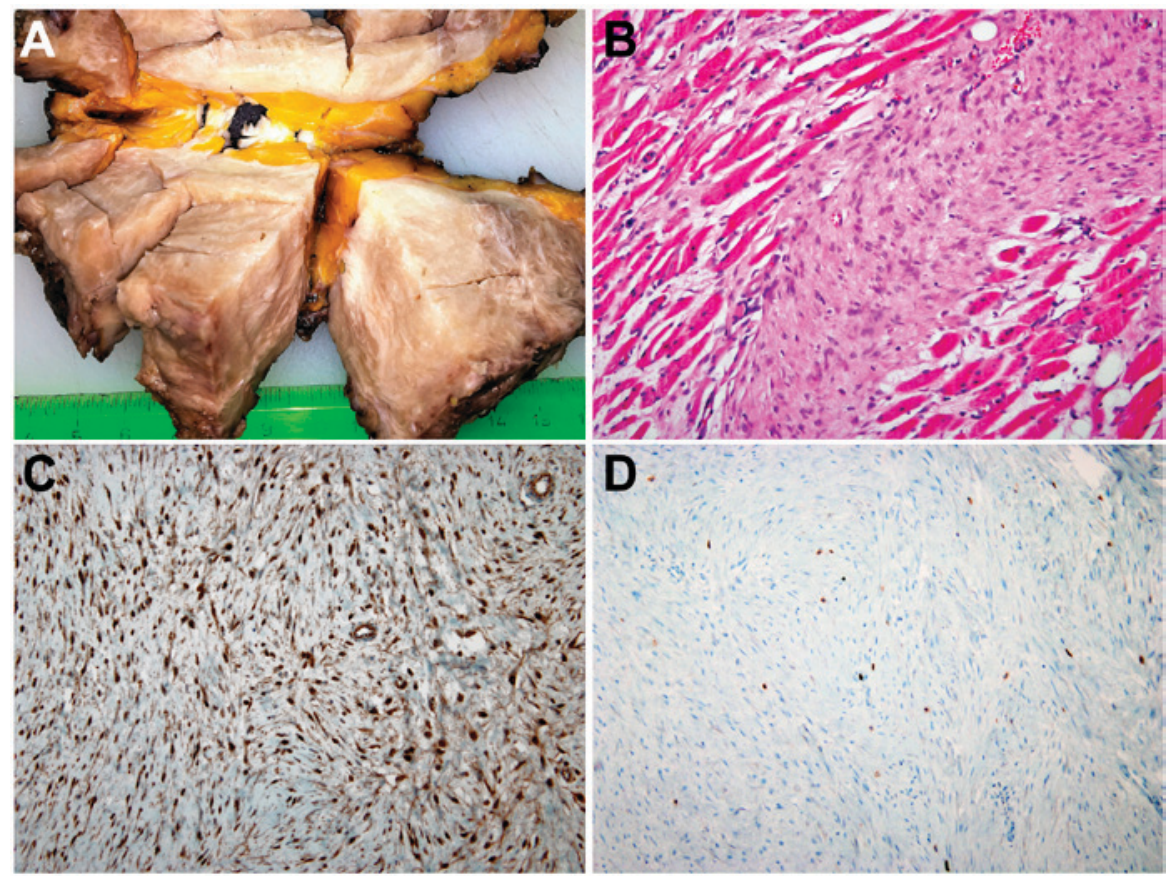

Figure 4. Breast fibromatosis in case 1. (A) Poorly circumscribed tumor with infiltration of the adjacent tissue, including muscle, in gross examination. (B) A predominant spindle cell proliferation of bipolar fibroblasts and myofibroblasts with low mitotic activity, without cell atypia upon hematoxylin and eosin staining (magnification, x200). (C) Upon immunohistochemical staining (magnification, x200), diffuse nuclear $\beta$-catenin positivity in fibromatous proliferation was present. (D) In total, $<5 \%$ of the spindle cells were positive for Ki-67 (MIB1).

RM2235, Leica Biosystems GmbH, Nussloch, Germany) to produce $5-\mu \mathrm{m}$ sections. The sections were then stained with hematoxylin and eosin.

Histological evaluation of the surgical specimens revealed a proliferation of more or less evenly spaced uniform spindle cells arranged in intersecting fascicles and associated with mild to moderate amounts of collagen and occasional mitotic figures (Fig. 4B). The tumor invasion of the underlying tissue involved resected muscles and inter-/pericostal soft tissue. There was no evidence of invasion into the bony ribs or underlying parietal pleura. A definitive diagnosis of breast desmoid-type fibromatosis was established from serial paraffin sectioning (as previously described) and immunohistochemistry (IHC). IHC analysis of the tumor with anti- $\beta$-catenin antibody (rabbit primary monoclonal antibody; clone E247; catalog no. AB32572; dilution, 1:200; incubation for $60 \mathrm{~min}$ at $25^{\circ} \mathrm{C}$; Abcam, Cambridge, UK) revealed diffuse nuclear positivity (Fig. 4C). The Ki-67 proliferation index (defined as the percentage of the total number of tumor cells with nuclear staining) measured on paraffin sections using the MIB-1 antibody (rabbit monoclonal antibody; clone MIB-1; catalog no. M7240; dilution, 1:100; incubation for $30 \mathrm{~min}$ at $25^{\circ} \mathrm{C}$; Dako; Agilent Technologies, Inc., Santa Clara, CA, USA) was $<5 \%$ (Fig. 4D). The post-operative course was uneventful and the patient was discharged home on post-operative day 7. The patient is now 10 months post-surgery and remains disease-free.

Case 2. A 43-year-old, premenopausal Caucasian woman with no previous medical history was referred in October 2016 to 


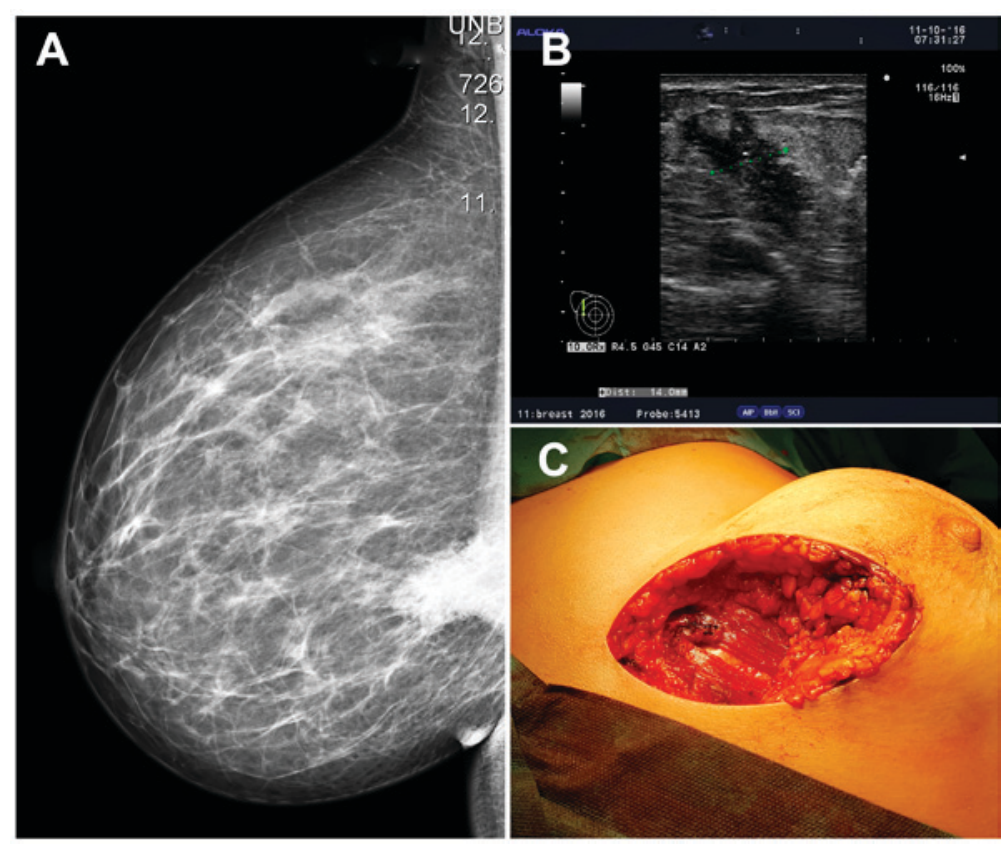

Figure 5. Breast fibromatosis in case 2. (A) Mammographic and (B) ultrasound examinations suggested an invasive breast tumor, probably carcinoma, infiltrating the muscles of the chest wall. (C) The patient underwent a quadrantectomy with a partial resection of the underlying musculature.
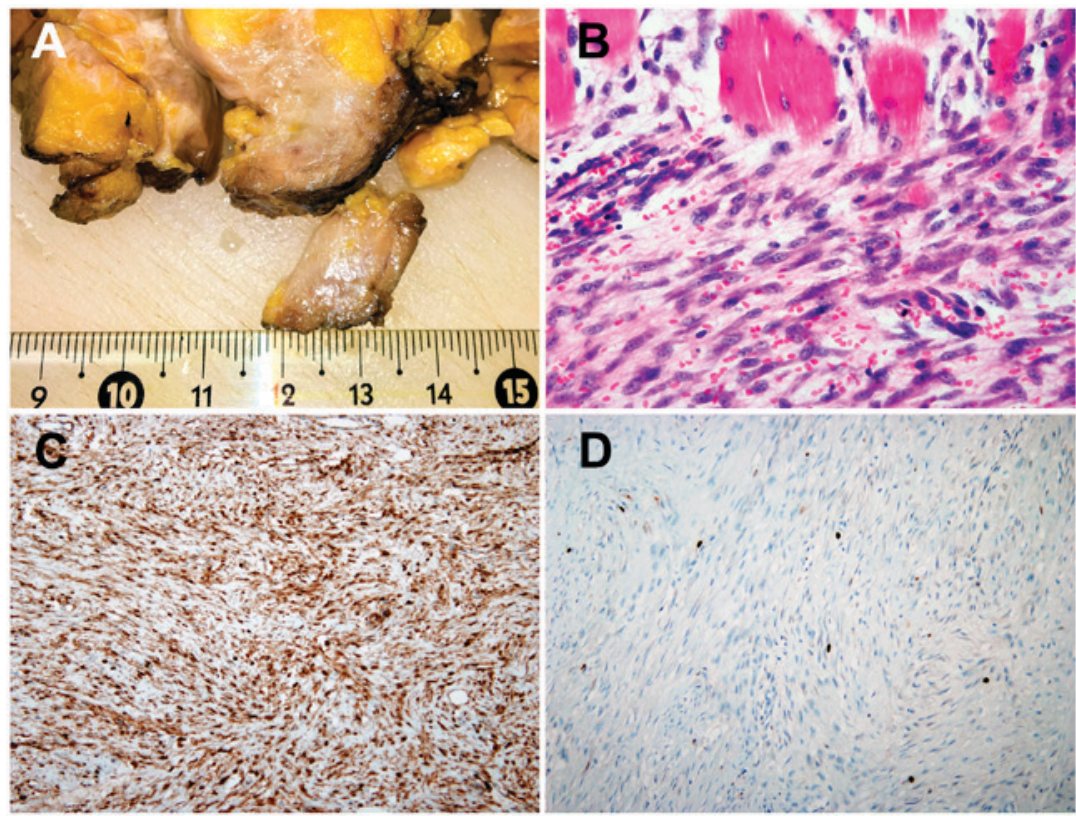

Figure 6. Breast fibromatosis in case 2. (A) Gross sectional view of the pathology specimen. (B) High-power histological view showing fibromatosis invading into the adjacent skeletal muscle (hematoxylin and eosin; magnification, $\mathrm{x} 400$ ). (C) Diffuse nuclear $\beta$-catenin positivity and (D) low Ki-67 (MIB1) positivity in fibromatous proliferation (magnification, $\mathrm{x} 200$ ).

the Breast Unit of the Second Department of Gynecology and Obstetrics, University Hospital of Bratislava, for a palpable lump in the inner, lower side of the right breast, which was fixed to the chest wall. Breast imaging examinations suggested an invasive breast tumor, likely carcinoma, infiltrating the muscles of the chest wall (Fig. 5A and B). Therefore, the patient underwent a thoracic computed tomography scan, which showed an obscured chest wall infiltration. An ultrasound-guided core needle biopsy revealed a low-grade myofibroblastic proliferation consistent with breast fibromatosis. As in case 1, the patient was transferred to an operating room in the Department of Thoracic
Surgery (University Hospital of Bratislava) for breast saving surgery and possible en bloc resection of the underlying regions of the thoracic wall. Surgery was performed under general anesthesia with selective intubation. The patient underwent a right quadrantectomy, with a partial resection of the underlying musculature (Fig. 5C). A definitive diagnosis of breast fibromatosis was established from serial paraffin sectioning and immunohistochemistry (using the aforementioned IHC procedures from case 1; Fig. 6). The spindle cell proliferations invaded only into the adjacent skeletal muscle. The patient is now 8 months post-surgery and remains disease-free. 


\section{Discussion}

The World Health Organization defines desmoid-type fibromatosis as an intermediate soft-tissue tumor that is characterized by clonal fibroblastic proliferation arising in the deep soft tissues, with a tendency for infiltration of the local tissues and local recurrence, but an inability to metastasize (1). Cases of desmoid-type fibromatosis are often broadly categorized into one of two groups. The largest group contains sporadic cases, with an incidence of 3.42 per million person-years, forming $84-93 \%$ of all cases $(1,6)$. In this group, somatic $\beta$-catenin-activating mutations are considered to be the cause of the disease (13). The second, smaller group of cases consists of those associated with familial adenomatous polyposis (14). The etiology of mammary fibromatosis is unknown; it has no predilection for age, family history or exposure factors, although certain cases occur after trauma $(4,6)$. The disease is usually painless and the presenting symptom is always a palpable, firm breast mass $(2,6,7)$. Breast imaging examinations are not specific for fibromatosis and often imitate breast cancer $(8,11)$ (Fig. 5A and B). Computed tomography and/or magnetic resonance imaging assists in defining the infiltration into adjacent tissue, particularly in patients in whom there was a preoperative suspicion of chest wall musculature involvement (1,2,11; Fig. 2). Large core-needle biopsies are not always successful in the differential diagnosis of tumors of mesenchymal origin (15). A diagnosis can be made from microscopic findings on routine hematoxylin and eosin-stained sections. In general, the lesion does not have malignant features such as a high mitotic rate, cellular atypia, necrosis or vascular invasion $(15,16)$. Immunohistochemical staining for $\beta$-catenin with nuclear positivity is also useful in establishing a diagnosis (Figs. 4C and 6C), but there are no specific immunomarkers for breast fibromatosis $(1,15)$.

Standard treatment of this recurrent tumor involves a wide surgical resection with safe margins (1-5). The reasons for such an aggressive surgical approach include the potential for the fibromatosis to undergo aggressive, local growth and for invasion into the surrounding structures, plus a high local recurrence rate when incompletely excised with positive surgical margins. Povoski et al (2) described the requirement for repeated surgical intervention in a patient with multiple recurrences of fibromatosis due to positive resection margins in specimens from the primary surgery. The surgical management throughout the final surgical treatment of the disease, which involved infiltration of the chest wall, was very similar to the procedure in case 1 of the present study (Fig. 3A and B). In the present study, a wide en bloc resection was chosen to avoid future recurrence, based on negative surgical margins on the frozen section as an alternative to mastectomy. Mastectomy should be avoided in cases where it is possible, particularly in young women $(1,16,17)$. Ha et al (8) presented the case of a 30 -year-old Caucasian woman with a palpable mass within the medial portion of the right breast. The mammographic presentation of breast fibromatosis mimicking breast carcinoma was almost identical to that of case 2 in the present study (Fig. 5A). The lesion was surgically resected via a wide local excision. Follow-up mammograms that were performed at 1 and 2 years post-resection showed no evidence of recurrence on radiography (8). Notably, 24 cases of breast desmoid tumors that developed following augmentation mammoplasty have been described in the literature (12). In total, 16 of the patients developed fibromatosis in association with silicone implants. The natural progression of fibromatosis remains unpredictable and enigmatic. While this disease has been described as progressing rapidly and aggressively in certain cases, in other cases, desmoid tumors have demonstrated slow and locally invasive growth $(1,7,15)$. Several potential characteristics (including tumor size, location and patient age) have been investigated as predictors of tumor behavior, but the specific parameters of these predictors are yet to be determined $(1,17)$.

A reassessment of the overall management of fibromatosis has taken place over the last few years, and preservation of function has become a priority (17-22). A biopsy confirming diagnosis is mandatory and should be confirmed by an expert pathologist. Immunostaining for $\beta$-catenin with nuclear positivity is useful in establishing a diagnosis (15). Active surveillance has only been shown to lead to spontaneous regression of $28-50 \%$ of cases of extra-abdominal fibromatosis $(18,22)$. Surgery remains a valid option, however, preservation of function and quality of life are essential $(16,17,21)$. The role of adjuvant radiotherapy also remains unclear, and the optimal regimes, doses and durations of other systemic therapies (including chemotherapy, hormonal therapy and tyrosine kinase inhibitors) require elucidation $(1,16,22)$. Patients should be followed closely for at least 3 years to monitor regression or recurrence (16). Magnetic resonance scans are best suited to accurately reflect disease progression or regression, or to assess treatment response.

In conclusion, desmoid-like fibromatosis is a rare breast neoplasm. Despite its classification as an intermediate soft-tissue tumor, breast fibromatosis does possess the potential for aggressive local behavior. Breast imaging examinations are not specific for fibromatosis and often imitate breast cancer. Surgery remains a valid option, however, preservation of function and quality of life are essential. The role of adjuvant therapy is also not entirely clear, and the optimal regimes, doses and durations of systemic treatment of the disease require elucidation. Due to the rare involvement of the breast in patients with desmoid-like fibromatosis, the present study reports 2 cases with their clinical features and histological findings in order to improve and add to our knowledge of the disease.

\section{References}

1. Eastley N, McCulloch T, Esler C, Hennig I, Fairbairn J, Gronchi A and Ashford R: Extra-abdominal desmoid fibromatosis: A review of management, current guidance and unanswered questions. Eur J Surg Oncol 42: 1071-1083, 2016.

2. Povoski SP, Marsh WL Jr, Spigos DG, Abbas EA and Buchele BA: Management of a patient with multiple recurrences of fibromatosis (desmoid tumor) of the breast involving the chest wall musculature. World J Surg Oncol 4: 32, 2006.

3. Shen C, Zhou Y and Che G: Management of a female with recurrence of fibromatosis of the chest wall adjacent to the breast: A case report. J Cardiothorac Surg 8: 41, 2013.

4. Abbas AE, Deschamps C, Cassivi SD, Nichols FC III, Allen MS, Schleck CD and Pairolero PC: Chest-wall desmoid tumors: Results of surgical intervention. Ann Thorac Surg 78: 1219-1223, 2004.

5. Croce S, Letourneux C, Dale G and Mathelin C: Breast fibromatosis: An uncommon benign breast disease. Gynecol Obstet Fertil 37: 442-446, 2009.

6. Taylor TV and Sosa J. Bilateral breast fibromatosis: Case report and review of the literature. J Surg Educ 68: 320-325, 2011. 
7. Yamaguchi H, Sakakibara T, Hino M, Ryu M, Senuma K Nakai K, Tomiki Y, Sakamoto K, Kamano T, Tsurumaru M and Matsumoto T: A case of fibromatosis of the breast. Breast Cancer 9: 175-178, 2002.

8. Ha KY, Deleon P and Hamilton R: Breast fibromatosis mimicking breast carcinoma. Proc (Bayl Univ Med Cent) 26: 22-24, 2013.

9. He P, Cui LG, Lei YT, Liu JY and Wang JR: Ultrasound elastographic findings of mammary fibromatosis. Case Rep Radiol 2015: 829468, 2015.

10. Plaza MJ and Yepes M: Breast fibromatosis response to tamoxifen: Dynamic MRI findings and review of the current treatment options. J Radiol Case Rep 6: 16-23, 2012.

11. Matherne TH, Green A Jr, Tucker JA and Dyess DL: Fibromatosis: The breast cancer imitator. South Med J 97: 1100-1103, 2004.

12. Jeong WS, Oh TS, Sim HB and Eom JS: Desmoid tumor following augmentation mammoplasty with silicone implants. Arch Plast Surg 40: 470-472, 2013.

13. Hamada S, Futamura N, Ikuta K, Urakawa H, Kozawa E, Ishiguro $\mathrm{N}$ and Nishida Y: CTNNB1 S45F mutation predicts poor efficacy of meloxicam treatment for desmoid tumors: A pilot study. PLoS One 9: e96391, 2014.

14. Nieuwenhuis MH, Casparie M, Mathus-Vliegen LM, Dekkers OM, Hogendoorn PCW and Vasen HF: A nation-wide study comparing sporadicand familial adenomatous polyposis-related desmoid-type fibromatoses. Int J Cancer 129: 256-261, 2011.

15. Rakha EA, Badve S, Eusebi V, Reis-Filho JS, Fox SB, Dabbs DJ, Decker T, Hodi Z, Ichihara S, Lee AH, et al: Breast lesions of uncertain malignant nature and limited metastatic potential: Proposals to improve their recognition and clinical management. Histopathology 68: 45-56, 2016.

16. Kasper B, Baumgarten C, Bonvalot S, Haas R, Haller F, Hohenberger P, Moreau G, van der Graaf WT and Gronchi A; Desmoid Working Group: Management of sporadic desmoid-type fibromatosis: a European consensus approach based on patients and professionals' expertise-a sarcoma patients EuroNet and European Organisation for Research and Treatment of Cancer/Soft Tissue and Bone Sarcoma Group initiative. Eur J Cancer 51: 127-136, 2015.
17. Fiore M, Rimareix F, Mariani L, Domont J, Collini P, Le Péchoux C, Casali PG, Le Cesne A, Gronchi A and Bonvalot S: Desmoid-type fibromatosis: A front-line conservative approach to select patients for surgical treatment. Ann Surg Oncol 16: 2587-2593, 2009.

18. Bonvalot $\mathrm{S}$, Ternès $\mathrm{N}$, Fiore $\mathrm{M}$, Bitsakou $\mathrm{G}$, Colombo $\mathrm{C}$, Honoré C, Marrari A, Le Cesne A, Perrone F, Dunant A and Gronchi A: Spontaneous regression of primary abdominal wall desmoid tumors: More common than previously thought. Ann Surg Oncol 20: 4096-4102, 2013.

19. Crago AM, Denton B, Salas S, Dufresne A, Mezhir JJ, Hameed M, Gonen M, Singer S and Brennan MF: A prognostic nomogram for prediction of recurrence in desmoid fibromatosis. Ann Surg 258: 347-353, 2013.

20. Keus RB, Nout RA, Blay JY, de Jong JM, Hennig I, Saran F, Hartmann JT, Sunyach MP, Gwyther SJ, Ouali M, et al: Results of a phase II pilot study of moderate dose radiotherapy for inoperable desmoid-type fibromatosis-an EORTC STBSG and ROG study (EORTC 62991-22998). Ann Oncol 24: 2672-2676, 2013.

21. Colombo C, Miceli R, Le Péchoux C, Palassini E, Honoré C, Stacchiotti S, Mir O, Casali PG, Dômont J, Fiore M, et al: Sporadic extra abdominal wall desmoid-type fibromatosis: Surgical resection can be safely limited to a minority of patients. Eur J Cancer 51: 186-192, 2015.

22. Al-Jazrawe M, Au M and Alman B: Optimal therapy for desmoid tumors: Current options and challenges for the future. Expert Rev Anticancer Ther 15: 1443-1458, 2015. 